\title{
SEMIPERFECT MIN-CS RINGS
}

\author{
JOSÉ L. GÓMEZ PARDO \\ Departamento de Alxebra, Universidade de Santiago, 15771 Santiago de Compostela, Spain \\ and MOHAMED F. YOUSIF \\ Department of Mathematics, Ohio State University, Lima, Ohio 45804, USA
}

(Received 1 July, 1997; revised 15 June 1998)

\begin{abstract}
We show that if $R$ is a ring such that each minimal left ideal is essential in a (direct) summand of ${ }_{R} R$, then the dual of each simple right $R$-module is simple if and only if $R$ is semiperfect with $\operatorname{Soc}\left({ }_{R} R\right)=\operatorname{Soc}\left(R_{R}\right)$ and $\operatorname{Soc}(R e)$ is simple and essential for every local idempotent $e$ of $R$. We also show that $R$ is left CS and right Kasch if and only if $R$ is a semiperfect left continuous ring with $\operatorname{Soc}\left(R_{R}\right) \subseteq{ }^{e}{ }_{R} R$. As a particular case of both results we obtain that $R$ is a ring such that every (essential) closure of a minimal left ideal is summand ( $R$ is then said to be left strongly min-CS) and the dual of each simple right $R$-module is simple if and only if $R$ is a semiperfect left continuous ring with $\operatorname{Soc}\left({ }_{R} R\right)=\operatorname{Soc}\left(R_{R}\right) \subseteq{ }_{R} R$. Moreover, in this case $R$ is also left $\operatorname{Kasch}, \operatorname{Soc}(e R) \neq 0$ for every local idempotent $e$ of $R$, and $R$ admits a (Nakayama) permutation of a basic set of primitive idempotents. As a consequence of this result we characterise left PF rings in terms of simple modules over the $2 \times 2$ matrix ring by showing that $R$ is left PF if and only if $M_{2}(R)$ is a left strongly min-CS ring such that the dual of every simple right module is simple.
\end{abstract}

1. Introduction. An important source of semiperfect rings is given by the theorem of B. Osofsky [14] which asserts that a left injective cogenerator ring (also called a left PF ring) is semiperfect and has finitely generated essential left socle. Conversely, if $R$ is left self-injective, semiperfect, and has essential left socle, then $R$ is left PF [15, 48.12]. It is obvious that $R$ is a left PF ring if and only if it is left selfinjective and left Kasch, where the latter condition just means that every simple left $R$-module is isomorphic to a (minimal) left ideal. From Osofsky's theorem it also follows that a left PF ring is right Kasch and so it is natural to ask whether a left self-injective right Kasch ring is left PF. This problem is still open but in order to obtain a positive solution it would be enough to prove that $R$ has essential socle, because it has already been shown in [6] that these rings are semiperfect. This result was extended in [18], where it was shown that if $R$ is left CS and the dual of every simple right $R$-module is simple, then $R$ is semiperfect with $\operatorname{Soc}\left({ }_{R} R\right)=\operatorname{Soc}\left(R_{R}\right) \subseteq{ }^{e}{ }_{R} R$.

In this paper we look for the weakest conditions of this type that imply the ring is semiperfect. Instead of left CS rings we consider the much larger class of left minCS rings (cf. [13]), i.e., rings $R$ such that every minimal left ideal is essential in a direct summand and we show that this weak injectivity property is useful to obtain semiperfect rings. Indeed, we prove in Theorem 2.1 that if $R$ is left min-CS, then the dual of every simple right $R$-module is simple if and only if $R$ is semiperfect with $\operatorname{Soc}\left({ }_{R} R\right)=\operatorname{Soc}\left(R_{R}\right)$ and $\operatorname{Soc}(R e)$ is simple and essential for every local idempotent $e$ of $R$. Thus we establish the following pattern: we work with an injectivity condition 
on the left and a "cogenerating" condition on the right, both closely related to simple modules, and we try to prove that $R$ is semiperfect and, in some cases, that $R$ is in a certain sense close to being left PF. The hypotheses of Theorem 2.1 (see also Theorem 2.2) are the weakest known conditions of this type that imply that $R$ is semiperfect.

If we replace the left min-CS condition used in Theorem 2.1 by the stronger one requiring that each closed left ideal with simple essential socle be a direct summand of ${ }_{R} R$ (we will then say that $R$ is left strongly min-CS), we obtain a class of rings that satisfies many of the characteristic properties of left PF rings. Thus we show in Theorem 2.4 that $R$ is left strongly min-CS and the dual of every simple right $R$ module is simple if and only if $R$ is a semiperfect left continuous ring with $\operatorname{Soc}\left({ }_{R} R\right)=\operatorname{Soc}\left(R_{R}\right)$ and $\operatorname{Soc}(R e)$ is simple and essential in $R e$ for every local idempotent $e$ of $R$. Furthermore, in this case $R$ is also left $\operatorname{Kasch,~} \operatorname{Soc}(e R) \neq 0$ for every local idempotent $e$ of $R$, and $R$ admits a (Nakayama) permutation of a basic set of primitive idempotents.

If instead of assuming that the duals of simple right $R$-modules are simple we suppose, more generally, that $R$ is right Kasch, then we obtain a larger class of rings that still retains many of these properties, for we show in Theorem 2.2 that $R$ is left CS and right Kasch if and only if it is semiperfect and left continuous with $\operatorname{Soc}\left(R_{R}\right) \subseteq{ }_{R} R$. In contrast with this result it is perhaps worth mentioning that while it has been shown in [7] that every left CS left Kasch ring has finitely generated essential left socle, it is still unknown whether these rings must be semiperfect. On the other hand, as an immediate consequence of our work we obtain a new characterisation of left PF rings in terms of simple modules by showing in Corollary 2.6 that $R$ is left PF if and only if the ring $S=M_{2}(R)$ of $2 \times 2$-matrices over $R$ is left strongly min-CS and the dual of every simple right $S$-module is simple.

Throughout this paper all rings $R$ will be associative and with identity and all modules are unitary $R$-modules. We will write $M_{R}$ to emphasise the fact that $M$ is a right $R$-module and, similarly, ${ }_{R} N$ will denote a left $R$-module.

We write $M \subseteq N(M \subset N)$ to mean that $M$ is a (proper) submodule of $N$ and $M \subseteq \subseteq^{e} N$ indicate that $M$ is an essential submodule of $N$. If $M$ is a right $R$-module, we will denote by $\operatorname{Soc}(M)$ the socle of $M$. The left (resp. right) annihilator of a subset $X$ of $R$ is denoted by $l(X)$ (resp. $r(X)$ ). The (Jacobson) radical of $R$ will be denoted by $J$.

A module $M_{R}$ is said to satisfy the C1-condition (or CS-condition) (resp. the min-CS condition) whenever every submodule (resp. simple submodule) of $M$ is essential in a direct summand of $M . M$ satisfies the C2-condition when every submodule of $M$ which is isomorphic to a direct summand of $M$ is itself a direct summand of $M . M$ is called continuous if it satisfies both the $\mathrm{C} 1-$ and the $\mathrm{C} 2$-conditions.

The ring $R$ is called right CS when $R_{R}$ is a CS module, and similarly for the other conditions we have just defined for modules. $R$ is said to be a right Kasch ring when every simple right $R$-module embeds in $R_{R} . R$ is called right mininjective [13] if every $R$-homomorphism from a minimal right ideal of $R$ into $R$ is given by left multiplication by an element of $R$. By [13, Proposition 2.2], the dual of every simple right $R$-module is simple if and only if $R$ is a right Kasch right mininjective ring.

We refer to $[\mathbf{1 0 , 1 5}]$ for all undefined notions used in the text.

2. Results We begin by looking at the structure of left min-CS right Kasch rings. 
THEOREM 2.1 Let $R$ be a left min-CS ring such that the dual of every simple right $R$-module is simple. Then the following statements hold:

(i) $R$ is semiperfect

(ii) For every $x \in R, R x$ is a minimal left ideal if and only if $x R$ is a minimal right ideal. In particular, $\operatorname{Soc}\left({ }_{R} R\right)=\operatorname{Soc}\left(R_{R}\right)$.

(iii) Every minimal left ideal of $R$ is an annihilator.

(iv) $\operatorname{Soc}(R e)$ is simple and essential in Re, for every local idempotent e of $R$. In particular, $\operatorname{Soc}(R) \subseteq{ }^{e} R$ and $R$ is left finite-dimensional.

(v) $R$ is left Kasch if and only if $\operatorname{Soc}(e R) \neq 0$ for every local idempotent e of $R$. Moreover, in this case the following assertions hold:

(a) $\operatorname{Soc}(e R)$ is homogenous for every local idempotent e of $R$.

(b) If $\left\{e_{1}, \ldots, e_{n}\right\}$ is a basic set of primitive orthogonal idempotents, then there exist elements $x_{1}, \ldots, x_{n}$ in $R$ and a (Nakayama) permutation $\sigma$ of $\{1, \ldots, n\}$ such that the following hold for each $i=1, \ldots, n$ :

$x_{i} R \subseteq \operatorname{Soc}\left(e_{i} R\right)$.

$R x_{i}=\operatorname{Soc}\left(R e_{\sigma_{i}}\right)$ is simple and essential in $R e_{\sigma_{i}}$.

$x_{i} R \cong e_{\sigma_{i}} R / e_{\sigma_{i}} J, R x_{i} \cong R e_{i} / J e_{i}$.

$\left\{x_{1} R, \ldots, x_{n} R\right\}$ and $\left\{R x_{1}, \ldots, R x_{n}\right\}$ are complete sets of representatives of the isomorphism classes of simple right and left $R$-modules, respectively.

Conversely, if $R$ is a semiperfect ring with $\operatorname{Soc}\left({ }_{R} R\right)=\operatorname{Soc}\left(R_{R}\right)$, and $\operatorname{Soc}(R e)$ is simple for every local idempotent e of $R$, then the dual of every simple right $R$-module is simple.

Proof. (i) We show that every simple right $R$-module has a projective cover. Let $C$ be a simple right $R$-module and $M$ a maximal right ideal of $R$ such that $C \cong R / M$. Then $l(M) \cong C^{*}=\operatorname{Hom}_{R}\left(C_{R}, R_{R}\right)$ is simple by hypothesis and so there exists an idempotent element $e \in R$ such that $l(M) \subseteq$ Re. Observe that $e R$ is not contained in $M$, for if $e \in M$, then $l(M) \cdot e=0$ and so $l(M)=0$, a contradiction. Since $M$ is maximal in $R_{R}$ we have that $e R+M=R$ and so $e R /(e R \cap M) \cong$ $(e R+M) / M \cong R / M \cong C$. Thus it suffices to show that $e R \cap M$ is a small submodule of $e R$. Let $L$ be a maximal submodule of $e R$ such that $L+(e R \cap M)=e R$. Since $e R / L \cong R /((1-e) R+L)$, we have that $(e R / L)^{*} \cong l((1-e) R+L)=\operatorname{Re} \cap l(L)$. Then our hypotheses imply that $\operatorname{Re} \cap l(L) \neq 0$, because it is isomorphic to the dual of the simple right $R$-module $e R / L$. On the other hand, $L+(e R \cap M)=e R$ implies that $L$ is not contained in $M$ and, since, $M$ is maximal, that $L+M=R$. Thus we see that $l(L) \cap l(M)=0$ and so $(\operatorname{Re} \cap l(L)) \cap l(M)=0$. Since $l(M) \subseteq \subseteq^{e}$ Re it follows that $\operatorname{Re} \cap l(L)=0$. This gives a contradiction and shows that $e R \cap M$ is the unique maximal submodule of $e R$, so that $e R \cap M$ is small and $e R$ is indeed a projective cover of $C \cong R / M$.

(ii) By [13, Theorem 1.14], if $x R$ is a minimal right ideal of $R$, then $R x$ is a minimal left ideal of $R$. Conversely, suppose that $R x$ is a minimal left ideal of $R$. Since $R$ is a left min-CS-ring, $R x \subseteq^{e} R e$ for some idempotent $e \in R$ which is actually a local idempotent because $R$ is semiperfect. Now $r(x) \supseteq(1-e) R$ and hence $r(x) \subseteq J(R)+(1-e) R$, which is the unique maximal right ideal containing $(1-e) R$. This implies that $\operatorname{lr}(x) \supseteq l(J(R)+(1-e) R)$ which is a minimal left ideal of $R$ by hypothesis. On the other hand, $R x \subseteq^{e} R e$ implies $R x \subseteq^{e} \operatorname{lr}(x) \subseteq R e$ and so $R x=l(J(R)+(1-e) R)$. Thus $r(x)=r l(J(R)+(1-e) R)=J(R)+(1-e) R$ because $R$ is right Kasch, and so $x R$ is a minimal right ideal of $R$. 
(iii) If $R x$ is a minimal left ideal of $R$ with $R x \subseteq^{e} R e$ for some (local) idempotent $e$ of $R$, it follows from the proof of (ii) above that $R x=l(J(R)+(1-e) R)$ and hence $R x=\operatorname{lr}(x)$.

(iv) As we have already remarked, our hypotheses imply that $R$ is a right Kasch right mininjective ring. Then it follows from [13, Proposition 3.3] that if $e$ is a local idempotent, then $R e$ has simple socle. Let now $R=R e_{1} \oplus \ldots \oplus R e_{n}$, where the $e_{i}$ are local idempotents and let $C$ be the socle of $R e_{j}$. Since $R$ is left min-CS, there exists a direct summand $K$ of ${ }_{R} R$ such that $C$ is essential in $K$. The decomposition ${ }_{R} R=R e_{1} \oplus \ldots R e_{n}$ complements direct summands by [1, Theorem 27.12] and, since $0 \neq C \subseteq K \cap R e_{j}$ and $K$ is indecomposable, we have that $R=K \oplus\left(\oplus_{i \neq j} R e_{i}\right)$. Thus $K \cong R e_{j}$, showing that $R e_{j}$ has simple essential socle.

(v) Suppose that $R$ is left Kasch and $e$ is a local idempotent of $R$. Then $0 \neq(R e / J e)^{*} \cong e \cdot r(J)=e \cdot \operatorname{Soc}\left({ }_{R} R\right)=e \cdot \operatorname{Soc}\left(R_{R}\right)=\operatorname{Soc}(e R) . \quad$ Conversely, $\quad$ if $\operatorname{Soc}(e R) \neq 0$ for every local idempotent $e$ of $R$, then we may apply [13, Theorem 3.7] to deduce that $R$ is a left Kasch ring. Furthermore, (a) and (b) also follow from [13, Theorem 3.7].

Finally, for the converse, suppose that $R$ is semiperfect with $\operatorname{Soc}\left({ }_{R} R\right)=\operatorname{Soc}\left(R_{R}\right)$ and $\operatorname{Soc}(R e)$ is simple for every local idempotent $e$ of $R$. Let $C$ be a simple right $R$ module. Then $C \cong e R / e J$ for some local idempotent $e$ of $R$ and so $C^{*} \cong(e R / e J)^{*} \cong l(J) \cdot e=\operatorname{Soc}(R e)$ is simple.

It was proved in [6] that a left self-injective right Kasch ring is semiperfect. As we have already remarked, it is an open question whether the left self-injective right Kasch rings are left PF but a left CS right Kasch ring need not be left PF. However, in the next theorem we show that left CS right Kasch rings are semiperfect.

THEOREM 2.2 A ring $R$ is left $C S$ and right Kasch if and only if $R$ is a semiperfect left continuous ring with $\operatorname{Soc}\left(R_{R}\right) \subseteq{ }_{R} R$.

Proof. Assume that $R$ is a left CS and right Kasch ring. As in the proof of Theorem 2.1 (i), let $C$ be a simple right $R$-module and $M$ a maximal right ideal of $R$ such that $C \cong R / M$. Since $R$ is left $\mathrm{CS}$, there exists an idempotent element $e$ of $R$ such that $l(M) \subseteq \subseteq^{e} R$. Then the proof of Theorem 2.1 (i) shows that $C$ has a projective cover and hence $R$ is semiperfect. Furthermore, $R$ is left continuous by [17, Lemma 1.15]. Now, since $R$ is right $\operatorname{Kasch}, \operatorname{Soc}\left(R_{R}\right) \neq 0$, and by the left CS-condition $\operatorname{Soc}\left(R_{R}\right) \subseteq \subseteq^{e} R e$ for some idempotent $e$ of $R$. Thus $(1-e) R \subseteq r\left(\operatorname{Soc}\left(R_{R}\right)\right)$. But $r\left(\operatorname{Soc}\left(R_{R}\right)\right)=J(R)$ because $R$ is right Kasch, which is a contradiction unless $e=1$ and $\operatorname{Soc}\left(R_{R}\right) \subseteq{ }_{R} R$.

Conversely, suppose that $R$ is semiperfect with $\operatorname{Soc}\left(R_{R}\right) \subseteq{ }_{R}^{e} R$. If $M$ is a maximal right ideal of $R$, then $M=e R \oplus(M \cap(1-e) R)$, where $e \in R$ is an idempotent and $(M \cap(1-e) R) \subseteq J(R)$. Thus $l(M)=R(1-e) \cap l(M \cap(1-e) R)$. But $\operatorname{Soc}\left(R_{R}\right)=$ $l(J(R)) \subseteq l(M \cap(1-e) R)$ and hence $l(M \cap(1-e) R)$ is essential in ${ }_{R} R$. Thus $l(M) \neq 0$ and $R$ is right Kasch.

In the next corollary we characterise left CS two-sided Kasch rings.

COROLlary 2.3 Let $R$ be a ring. Then the following conditions are equivalent:

(i) $R$ is a left $C S$ left and right Kasch ring.

(ii) $R$ is a semiperfect left continuous ring with essential left socle. 
Moreover, if $R$ satisfies these conditions, then the following hold.

(a) $\operatorname{Soc}(R e)$ is simple and essential in $R e$, and $\operatorname{Soc}(e R) \neq 0$ for every local idempotent e of $R$.

(b) If $\left\{e_{1}, \ldots, e_{n}\right\}$ is a basic set of local idempotents in $R$, there exist elements $x_{1}, \ldots, x_{n}$ of $R$ and a permutation $\sigma$ of $\{1, \ldots, n\}$ such that the following hold for each $i=1, \ldots, n$ :

$x_{i} R \subseteq \operatorname{Soc}\left(e_{i} R\right)$, and $R x_{i}=\operatorname{Soc}\left(R e_{\sigma_{i}}\right)$.

$x_{i} R \cong e_{\sigma_{i}} R / e_{\sigma_{i}} J$, and $R x_{i} \cong R e_{i} / J e_{i}$.

$\left\{x_{1} R, \ldots, x_{n} R\right\}$ and $\left\{R x_{1}, \ldots, R x_{n}\right\}$ are complete sets of representatives of the isomorphism classes of simple right and left $R$-modules, respectively.

Proof. (i) $\Rightarrow$ (ii) $R$ is semiperfect and left continuous by Theorem 2.2. Since $R$ is also left Kasch, it follows from [13, Lemma 4.15] that $\operatorname{Soc}\left({ }_{R} R\right) \subseteq{ }^{e} R$.

(ii) $\Rightarrow$ (i) follows from [13, Lemma 4.16].

Now for the rest of the assertions, observe first that, since $\operatorname{Soc}(R e) \neq 0$ and $R e$ is a CS-module, we have that $\operatorname{Soc}(R e)$ is simple and essential in $R e$ for every local idempotent $e$ of $R$. This proves the first part of (a). On the other hand, by Theorem 2.2 we have that $\operatorname{Soc}\left(R_{R}\right) \subseteq{ }_{R} R$, and hence $\operatorname{Soc}\left({ }_{R} R\right) \subseteq{ }^{e} \operatorname{Soc}\left(R_{R}\right)$.

Now, if $e$ is a local idempotent of $R$, then since $R$ is left Kasch we have that $0 \neq(R e / J e)^{*} \neq e \cdot r(J)=e \cdot \operatorname{Soc}\left({ }_{R} R\right) \subseteq e \cdot \operatorname{Soc}\left(R_{R}\right)=\operatorname{Soc}(e R)$. This completes the proof of (a). Finally, (b) follows from [13, Theorem 4.17].

We will say that a module $M$ is strongly min-CS if every (essential) closure of a simple submodule of $M$ is a summand. Accordingly, $R$ will be called left strongly min-CS if ${ }_{R} R$ is strongly min-CS. Observe that by [3, Lemma 1.4, Lemma 1.9], a module with finitely generated essential socle is strongly min-CS if and only if it is CS. It is well known that the $\operatorname{ring} R=\left(\begin{array}{ll}T & T \\ O & T\end{array}\right)$, where $T=\mathbb{Z} / 4 \mathbb{Z}$, is a right artinian ring which is not right CS (see, e.g., [7]) and hence it is not right strongly min-CS. However, it is easily checked that this ring is right min-CS.

THEOREM 2.4 Let $R$ be a ring. Then the following conditions are equivalent.

(i) $R$ is left strongly min-CS and the dual of every simple right $R$-module is simple.

(ii) $R$ is a semiperfect left continuous ring such that $\operatorname{Soc}\left({ }_{R} R\right)=\operatorname{Soc}\left(R_{R}\right) \subseteq{ }_{R} R$.

Moreover, if $R$ satisfies these conditions, then the following hold.

(a) $R$ is left Kasch.

(b) $\operatorname{Soc}(R e)$ is simple and essential in Re and $\operatorname{Soc}(e R)$ is non-zero and homogenous, for every local idempotent e of $R$.

(c) $R$ admits a (Nakayama) permutation of any basic set of primitive idempotents as in (b) of Theorem 2.1.

Proof. (ii) $\Rightarrow$ (i) Clearly every left continuous ring is left strongly min-CS. Now, if $e$ is a local idempotent of $R$, then $R e$ is a left CS-module, because summands of CS-modules are again CS. Since $\operatorname{Soc}\left({ }_{R} R\right) \subseteq{ }^{e}{ }_{R} R$, we have also that $\operatorname{Soc}(R e) \subseteq \subseteq^{e} R e$. Now, if $C$ is a simple submodule of $R e$, then $C$ is essential in a summand of $R e$ and so it is in fact essential in $R e$, because $R e$ is indecomposable. Then it follows from Theorem 2.1 that the dual of every simple right $R$-module is simple. 
(i) $\Rightarrow$ (ii) It follows from Theorem 2.1 that $R$ is semiperfect and $\operatorname{Soc}\left({ }_{R} R\right)=$ $\operatorname{Soc}\left(R_{R}\right) \subseteq{ }^{e}{ }_{R} R$. Moreover, $R$ satisfies the left C2-condition by [17, Lemma 1.15] and $R$ is a left CS-ring by [3, Lemma 1.4]. Therefore $R$ is left continuous, completing the proof of (ii).

Next, in order to prove the rest of the assertions (a)-(c), it is enough to show that $R$ is left Kasch, for then we can use Theorem $2.1(\mathrm{v})$. We can write ${ }_{R} R=R e_{1} \oplus \ldots \oplus$ $R e_{n}$, where $\left\{e_{1}, \ldots, e_{n}\right\}$ is a complete set of orthogonal local idempotents of $R$ with $\operatorname{Soc}\left(R e_{i}\right)$ simple and essential in $R e_{i}$ for each $i=1, \ldots, n$. Since $R$ is left continuous, each $R e_{i}$ is $R e_{j}$-injective if $i \neq j, 1 \leq i, j \leq n$. If $\operatorname{Soc}\left(R e_{i}\right) \cong \operatorname{Soc}\left(R e_{j}\right)$, then $R e_{i} \cong R e_{j}$ for $i, j, \in\{1, \ldots, n\}$ and so if $\left\{e_{i_{1}}, \ldots, e_{i_{t}}\right\}$ is a basic set of primitive idempotents with $i_{1}, \ldots, i_{t} \in\{1, \ldots, n\}$, then $\left\{\operatorname{Soc}\left(R e_{i_{j}}\right) \mid 1 \leq j \leq t\right\}$ is a complete set of representatives of the isomorphism classes of simple left $R$-modules, and hence $R$ is left Kasch.

As a consequence of the preceding results we next show that for the rings of Theorems 2.2 and 2.1 being noetherian is equivalent to being artinian.

COROLlary 2.5 Let $R$ be a ring. Suppose that $R$ is either left $C S$ and right Kasch, or a left min-CS ring such that the dual of each simple right $R$-module is simple. Then $R$ is right (resp. left) noetherian if and only if it is right (left) artinian.

Proof. In both cases we know, using Theorems 2.2 and 2.1, that $R$ is a semiperfect ring such that $\operatorname{Soc}\left(R_{R}\right) \subseteq{ }_{R}^{e} R$. If $R$ is right noetherian then it follows from [9, Corollary 1.4] that $J$ is nilpotent and so $R$ is right artinian. If $R$ is left noetherian then $J$ is nilpotent by $[9$, Corollary 1.5] and hence $R$ is left artinian.

We remark that even a (two-sided) artinian ring that satisfies the conditions of the preceding corollary need not be QF. For example, consider the ring defined in [2, p. 70], which can be regarded as a trivial extension in the following way. Let $K$ be a field and $\sigma$ an isomorphism of $K$ into a proper subfield $L \subseteq K$ such that $[K: L]$ is finite. Consider $K$ as a $(K, K)$-bimodule where the left $K$-module structure is the natural one and the right $K$-module structure is given by the endomorphism $\sigma$ of $K$, that is, $x \cdot a=a^{\sigma} x$ for $a, x \in K$. Let $R$ be the trivial extension of $K$ by the bimodule ${ }_{K} K_{K}$, i.e., $R=K \oplus K$ as abelian group, with multiplication given by $(a, x)(b, y)=$ $\left(a b, a y+b^{\sigma} x\right)$ for $(a, x),(b, y) \in R$. Then $R$ is a (two-sided) artinian local ring which is left continuous and satisfies that the dual of each simple right $R$-module is simple. Furthermore, each left ideal of $R$ is an annihilator and, using the characterisation of Morita duality for trivial extensions given in [11, Theorem 10], it can even be shown that $R$ has both a left and a right Morita duality. However, $R$ is not QF and, in fact, it can be readily seen that $R$ is not right min-CS and the dual of the unique simple left $R$-module is not simple.

In the next corollary we exploit the preceding results to obtain a characterisation of left PF rings in terms of simple modules over the $2 \times 2$ matrix ring.

COROLlary 2.6 Let $R$ be a ring and $S=M_{2}(R)$. Then the following conditions are equivalent:

(i) $R$ is a left $P F$ ring

(ii) $S$ is a left strongly min-CS ring such that the dual of every simple right $S$ module is simple. 
Proof. By [13, Theorem 1.6, Proposition 2.2], the property that every simple right module has simple dual is Morita invariant. Thus $S$ has this property if and only if so does $R$. Consequently, the implication (i) $\Rightarrow$ (ii) is clear. Conversely, observe that if (ii) holds, then $S$ is left continuous by Theorem 2.4 and hence $R$ is left self-injective by the work of Utumi [16]. Since by Morita invariance, the dual of every simple right $R$-module is simple, it follows from Theorem 2.4 that $R$ is a semiperfect ring with essential left socle and it is well known that $R$ is then left PF.

COROLlary 2.7 Let $R$ be a commutative ring. Then the following conditions are equivalent.

(i) $R$ is a min-CS Kasch ring.

(ii) $R$ is a semiperfect continuous ring with essential socle.

Proof. The implication (ii) $\Rightarrow$ (i) follows from Theorem 2.4. Conversely, assume that (i) holds. Then $R$ is a mininjective ring by [13, Proposition 4.12], and so the dual of every simple $R$-module is simple. Then it follows from Theorem 2.1 that $R$ is semiperfect with essential socle, and $R$ satisfies the C2-condition by [17, Lemma 1.15]. Write $R=R e_{1} \oplus \ldots \oplus R e_{n}$, where $\left\{e_{1}, \ldots, e_{n}\right\}$ is a complete set of orthogonal local idempotents of $R$. Since $\operatorname{Soc}\left(R e_{i}\right)$ is simple and essential in $R e_{i}$ for each $i=1, \ldots, n$, each $R e_{i}$ is uniform and hence every ideal is essential in a direct summand. Thus $R$ is a continuous ring.

Acknowledgements. This research was started during a visit by the second author to the Universidade de Santiago in the summer of 1996 . He would like to gratefully acknowledge the financial support of the host institution and thank the members of the Departamento de Alxebra for the warm hospitality he received during his visit. Also, the first author was partially supported by DGES (PB960961-C02-01, Spain.).

\section{REFERENCES} 1974).

1. F. W. Anderson and K. R. Fuller, Rings and categories of modules (Springer-Verlag,

2. J. E. Björk, Rings satisfying certain chain conditions, J. Reine Angew. Math. 245 (1970), 63-73.

3. C. Celik, A. Harmanci and P. F. Smith, A generalization of CS-modules, Comm. Algebra 23 (14) (1995), 5445-5460.

4. C. Faith and P. Menal, A counter example to a conjecture of Johns, Proc. Amer. Math. Soc. 116 (1992), 21-26.

5. C. Faith and P. Menal, The structure of Johns rings, Proc. Amer. Math. Soc. 120 (1994), 1071-1081.

6. J. L. García Hernández and J. L. Gómez Pardo, Closed submodules of free modules over the endomorphism ring of a quasi-injective module, Comm. Algebra 16 (1988), 115-137.

7. J. L. Gómez Pardo and P. A. Guil Asensio, Rings with finite essential socle, Proc. Amer. Math. Soc. 125 (1997), 971-977.

8. B. Johns, Annihilator conditions in noetherian rings, J. Algebra 49 (1977), 222-224.

9. B. Johns, Chain conditions and nil ideals, J. Algebra 73 (1981), 287-294.

10. S. H. Mohamed and B. J. Müller, Continuous and discrete modules (Cambridge University Press, 1990).

11. B. J. Müller, On Morita duality, Canad. J. Math 21 (1969), 1338-1347.

12. W. K. Nicholson and M. F. Yousif, On a theorem of Camillo, Comm. Algebra 23 (1995), 5309-5314. 
13. W. K. Nicholson and M. F. Yousif, Mininjective rings, J. Algebra 187 (1997), 548578. 387.

14. B. L. Osofsky, A generalization of quasi-Frobenius rings, J. Algebra 4 (1966), 373-

15. R. Wisbauer, Foundations of module and ring theory (Gordon and Breach, 1991).

16. Y. Utumi, On continuous rings and self-injective rings, Trans. Amer. Math. Soc. 118 (1965), 158-173.

17. M. F. Yousif, On continuous rings, J. Algebra 191 (1997), 495-509.

18. M. F. Yousif, CS rings and Nakayama permuations, Comm. Algebra 25 (1997), $3787-3795$. 